\title{
Parametric Description of Skeleton Radial Function by Legendre Polynomials for Binary Images Comparison
}

\author{
Olesia Kushnir and Oleg Seredin \\ Tula State University, Tula, Russia \\ kushnir-olesya@rambler.ru, oseredin@yandex.ru
}

\begin{abstract}
A new approach for shape comparison based on skeleton matching is proposed. The skeleton of a binary image is encoded as a series of primitives (chain of primitives). Traditionally, a primitive is a pair of numbers, the first one is the length of the some edge and the second one is the angle between this and the neighbour edges. As a novelty we offer to calculate the Legendre polynomial coefficients to describe the width of shape and incorporate them as the third vector component into the primitive. The procedure of the alignment of two primitive chains is suggested and the pair-wise comparison function based on optimal alignment is built. Experiments with developed comparison function on the realworld dataset of medicinal leaves show that the results of classification are appropriate considering the difficulty of the task and disadvantages of the database.
\end{abstract}

Keywords: binary image, skeleton radial function, primitive sequence, pair-wise comparison function, Legendre polynomials.

\section{Introduction}

Shape description is an important task for computer vision problems which can be solved in several ways. One of them is a skeleton representation. A skeleton is constructed as a locus of centers of maximal circles inscribed into the shape 4]. The weakness of skeletonization methodology is direct comparison of two skeletons. There are some ways how to compare skeletons. All of them have merits and demerits. We propose the novel approach to building procedure for featureless pair-wise comparison of skeletons, i.e. binary images.

There are the three points of what we intend to do. First, in Section 3 we suggest the idea of encoding the skeleton (a specific list of nodes and edges) as a series of primitives. More often we use the term "chain of primitives". Each primitive contains information about specific characteristics of the corresponding edge of skeleton. In order to compare the chains of primitives we will build the procedure of pair-wise alignment as it is traditionally used for amino-acids chains in bioinformatics (by the analogy with Levenshtein edit distance). In Section 5 we offer to take into account the object width using parametric description of

A. Elmoataz et al. (Eds.): ICISP 2014, LNCS 8509, pp. 520-530, 2014.

(C) Springer International Publishing Switzerland 2014 
skeleton radial function by Legendre polynomials. Experiments with developed comparison function on the model and real-world dataset of medicinal leaves (see Section 4) will be presented in Sections 6 and 7.

\section{Related Work}

The shape comparison methods can be divided into two groups. The first group is based on skeletons and the second one - on figures contours. The main approaches in the first group are 1) morphological shape comparison built on mathematical morphology of Serra [22] and morphological spectrums of the shape proposed by Maragos [12. Vizilter suggests the method of computing the distance between two images based on their spectrums using locus of centers of maximal circles [24, 2) comparing shapes by matching their skeletons via tree edit distance [10],3) path similarity which depends on the shortest distance between all skeleton nodes [6],4) path similarity that stands on the shortest distance between skeleton end nodes 1, 5) plenty of methods using features calculated on skeleton (2D and 3D) for solving certain classification problem [2 25]. The review of 2),3) and 4) methods is given in [1].

In a group of comparison methods founded on figures contours we point out three different approaches. The first one is formed on calculating the Procrustes distance between two ordered sets of points in the shapes boundary 8]. The second approach is built on the comparison of polygonal figure of the boundary: the edges of the figure are approximated by straight line segments of fixed length, the sequence of angles between the segments is chosen as string representation. Such representations are then compared by edit distances [16]. The third method is the mechanical model to compare the external outlines of the images. It is assumed that the contours are made of wire. The transformation of the contour is done by deforming the wire and is characterized by mechanical work. The minimal work is regarded as a measure of distinction between images [1921.

All the methods have strengths and weaknesses depending on the application task. The skeletal methods of comparison are more appropriate, for example, for the datasets including images with sophisticated well-pronounced shape and shapes with occlusions [120]. If shapes differ only by their width or in case of rather simple convex and concave shapes, the methods based on figures contours can be more fruitful.

\section{Skeletons Comparison Based on Primitive Chains}

To describe the skeleton we start traversing it from some initial node anticlockwise. Writing down the length of the current edge and the angle between the current and the next edges we obtain the sequence (chain) of primitives 511. Hence, a primitive is a pair of numbers $\omega=\{l, \alpha\}$. The first one is the scaled length of the current edge. Scaling unit is the diameter of minimal circle circumscribed about the skeleton. The second component of the primitive is normalized (to $2 \pi$ ) angle between the current and the next edges. 
The main concept of skeleton comparison is an alignment of two primitive chains. The mechanism of alignment is the following. We have two chains of different (in general) lengths. The first one we call base chain $\mathbb{B}$ and the second one we call the reference chain $\mathbb{R}$. Let's denote the elements of the base chain as $b_{1}, \ldots, b_{N} \in \Omega$ and the elements of the reference chain as $r_{1}, \ldots, r_{K} \in \Omega$, where $\Omega$ is a set of primitives, $N$ - the number of elements in chain $\mathbb{B}, K$ - the number of elements in chain $\mathbb{R}$ :

$$
\begin{aligned}
& \mathbb{B}: b_{1}, \ldots, b_{N}=\left\{l_{1}, \alpha_{1}\right\}\left\{l_{2}, \alpha_{2}\right\} \ldots\left\{l_{N}, \alpha_{N}\right\} \in \Omega, \\
& \mathbb{R}: r_{1}, \ldots, r_{K}=\left\{l_{1}, \alpha_{1}\right\}\left\{l_{2}, \alpha_{2}\right\} \ldots\left\{l_{K}, \alpha_{K}\right\} \in \Omega .
\end{aligned}
$$

To have a possibility of using gaps in the alignment we should extend the set of primitives by special element $g={ }^{\prime \prime}{ }^{\prime \prime}, g \in \Omega$. So we obtain the extended reference chain $\mathbb{E}$ with length $\bar{K}$, which consists of elements $e_{1}, \ldots, e_{\bar{K}} \in \Omega$ :

$$
\mathbb{E}: r_{1}, g, r_{2}, g, \ldots, g, r_{K}=e_{1}, \ldots, e_{\bar{K}}=\left\{l_{1}, \alpha_{1}\right\}-\left\{l_{3}, \alpha_{3}\right\}-\ldots-\left\{l_{\bar{K}}, \alpha_{\bar{K}}\right\} \in \Omega .
$$

The alignment will be determined by the reference vector $\mathbf{z}=\left\{z_{t}\right\}, t=1, \ldots, N$, where $N$ - the number of elements in the base chain. The value of $z_{t} \in\{1, \ldots, \bar{K}\}$, where $\bar{K}$ - the number of elements in the extended reference chain $\mathbb{E}$, will determine the order number of the element in the extended reference chain to what the $t$-th element of the base chain has the reference. In the alignment each element of the base chain has the reference to one certain element of the extended reference chain and several elements of the base chain can have references to the same element of the extended reference chain. Some variant of the alignment gives us some certain reference vector $\mathbf{z}$.

It is obvious that the goal is to find the optimal alignment (vector $\hat{\mathbf{z}}$ ) which shows our understanding of the comparison model. In other words, we have to minimize some criterion $J(\mathbf{z})$ relative to parameter $\mathbf{z}$. Our criterion is a sum of two parts. The first part we call node function and the second one we call edge function [17:

$$
J(\mathbf{z})=\sum_{t=1}^{N} \psi_{t}\left(z_{t}\right)+\sum_{t=2}^{N} \gamma_{t}\left(z_{t-1}, z_{t}\right) .
$$

Node function reflects the difference between two corresponding primitives in the base and the extended reference chains. It quadratically penalizes this difference: $\psi_{t}\left(z_{t}\right)=\rho\left(\omega^{\prime}, \omega^{\prime \prime} z_{t}\right), t \in\{1, \ldots, N\}, z_{t} \in\{1, \ldots, \bar{K}\}$ where

$$
\rho\left(\omega^{\prime}, \omega^{\prime \prime}\right)=\left(l^{\prime}-l^{\prime \prime}\right)^{2}+\left(\alpha^{\prime}-\alpha^{\prime \prime}\right)^{2} .
$$

The edge function (3) supplies the mutual order in pairwise alignment and defines the set of reasonable constrains. Cross-reference is prohibited, that is the condition $z_{t} \leq z_{t+1}$ must be hold in all cases. Equality $z_{t}=z_{t+1}$ can take place in case of the references to the same gap. This situation is penalized by positive real parameter $c$. Subsequences of gaps of different length are also fined by values depending on $c$. The cases of breaking the above mentioned constrains are penalized by $\infty$ value: 


$$
\gamma_{t}\left(z_{t-1}, z_{t}\right)=\left\{\begin{array}{l}
\infty, \quad z_{t}<z_{t-1}, \\
\infty, \quad z_{t}=z_{t-1} \wedge e_{z_{t}} \neq g, \\
c, \quad z_{t}=z_{t-1} \wedge e_{z_{t}}=g, \\
0, \quad z_{t}-z_{t-1}=1 \wedge e_{z_{t}} \neq g \wedge e_{z_{t-1}} \neq g, \\
0.5, \quad z_{t}-z_{t-1}=1, \\
0, \quad z_{t}-z_{t-1}=2 \wedge e_{z_{t}} \neq g \wedge e_{z_{t-1}} \neq g, \\
0.5, \quad z_{t}-z_{t-1}=2, \\
\left(z_{t}-z_{t-1}\right) c, \quad t \in\{2, \ldots, N\} .
\end{array}\right.
$$

So, we have to minimize criterion (1). This task is solved by means of dynamic programming. Based on the achieved alignment we calculate the measure of dissimilarity between the two skeletons:

$$
D(\hat{\mathbf{z}})=\sum_{t=1}^{N} \rho\left(\omega_{t}^{\prime}, \omega^{\prime \prime} z_{t}\right)
$$

where $\rho\left(\omega^{\prime}, \omega^{\prime \prime}\right)=\left\{\begin{array}{l}c, \quad \omega^{\prime}=g \quad \vee \quad \omega^{\prime \prime}=g, \\ \left(l^{\prime}-l^{\prime \prime}\right)^{2}+\left(\alpha^{\prime}-\alpha^{\prime \prime}\right)^{2}, \text { otherwise, }\end{array}\right.$ $\hat{\mathbf{z}} \in\{1, \ldots, \bar{K}\}-$ optimal set of references minimizing criterion (1).

In Fig. 1 there is a pictorial explanation of traversing the two skeletons and alignment of the two corresponding chains. The difference in skeletons according to matching procedure is marked as bold green. The value of dissimilarity measure according to (4) is equal to $D(\hat{\mathbf{z}})=0.876(c=0.2)$.

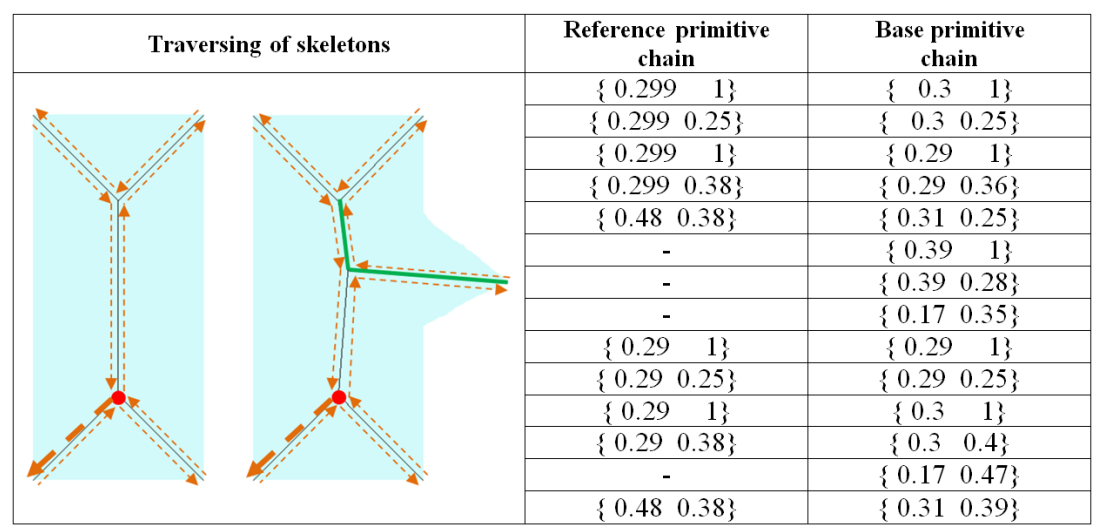

Fig. 1. The explanation of traversing of the two skeletons and alignment of the two corresponding primitive chains (thick point shows the initial position of traversing) 


\section{Materials Used as Real-World Application Task and Preliminary Results}

We use the application task of medicinal herbs classification as the starting point for research of the suggested dissimilarity function. This task is rather well-known in the related literature 37926. For example, some papers are devoted to the classification on the dataset "Leaves from Swedish Trees" [23].

The dataset we use for our research was brought to Moscow State University by Indian students. They had a practical task to build a procedure of classifying different kinds of herbs automatically for medicine purposes, using the information about shapes of different classes. There are 32 types of leaves represented by binary images in the dataset and the total number of objects is 1907 . So the average number of leaves in each class is near sixty. The smallest class has got 50 objects and the largest one - 77 objects. Examples from each class are in Fig. 2. We have to mention that the images of leaves were preprocessed via noise filtering.

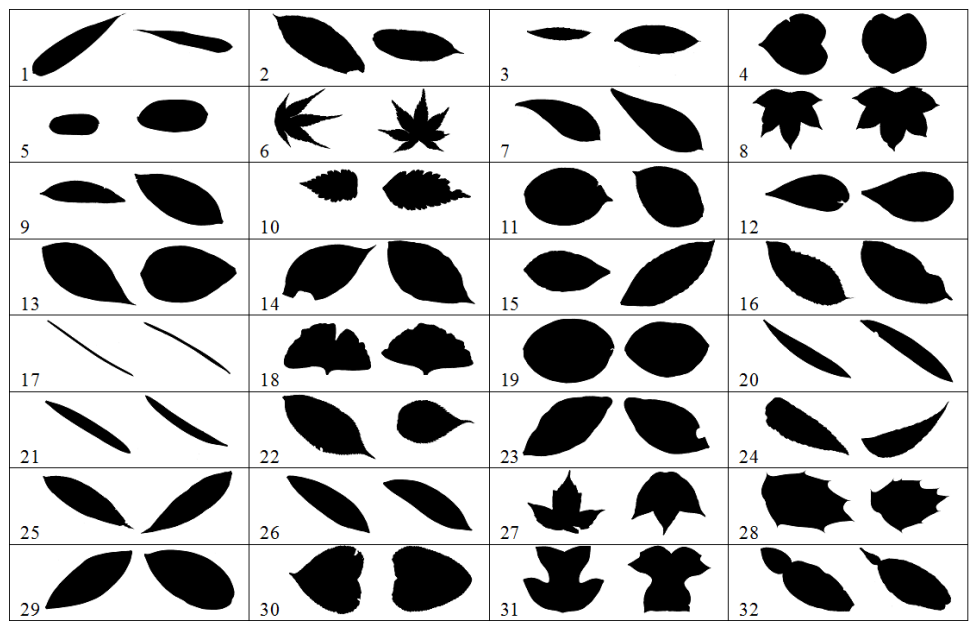

Fig. 2. Examples of classes in Medicinal Herbs Dataset

Let us notice that the dataset is rather difficult for classification: it contains large amount of classes, there are some classes which are very similar to each other and there are some classes with very different objects inside. So we have a multi-class recognition problem here with the dataset which practically hasn't been examined before. We intend to build our classifier and analyze recognition results in order to know, on the one hand, if our approach to shapes comparison works well, and on the other hand - if the dataset is built well.

We applied the comparison function (41) to the task of medicinal herbs classification. For the classes with pronounced skeleton topologies (see Fig. 3 a) the 


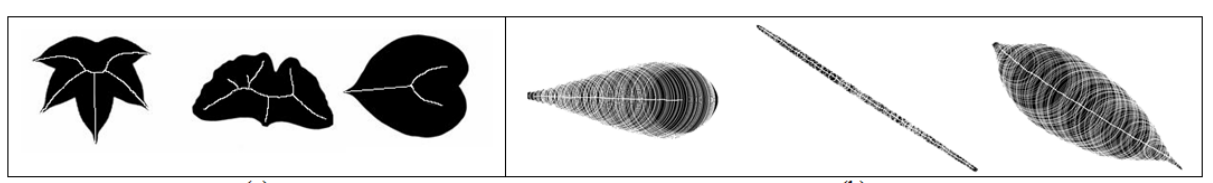

(a)

(b)

Fig. 3. (a) Species of leaves with pronounced skeleton topologies; (b) species of leaves with unrecognizable skeleton topologies

result was quite good. Rate of accuracy is $75-94 \%$. However, if the topology of skeletons is equal, such classes are unrecognizable (see Fig. 3b). Nevertheless, it is clear that the width profiles of such species are absolutely different.

To avoid described classification misbehavior we propose to take into account the radial function of skeleton in the comparison process. The radial function is determined as the value of radii of maximal inscribed circles with centers on each point of the skeleton. Thus, we suggest expanding a primitive by the information about radial function of skeleton. It will lead to an extra component in the node function (2) as well.

\section{Description of the Width of Object by Legendre Polynomials}

Let us notice that the initial skeleton of images with equal skeleton topology was a sequence of small ribs because of the construction. Details of skeletonization can be found in [13. As shown in Fig. 3b there are many circles with vertices on the ends of these ribs and they all contain exact information about the width of skeleton. For the comparison task we approximate this complicated skeleton replacing it by one straight edge. After approximation we lose all the information about width except for width in nodes of the edge.

To take the width of the object (in other words, the radial function of skeleton) into account in classification task we should use the information about the skeleton width before approximation. We propose to do this in the following way.

We have a binary image with skeleton before the approximation process (Fig. 4a). That is, we know the values of radius function in some nodes of the skeleton branch (Fig. 4 $4 \mathrm{~b}, \mathrm{c}$ ). Thus, we can interpolate the radius function using this information. To do this we suggest applying the Legendre polynomials. These polynomials are based on Legendre functions which are orthogonal to each other in the interval $[-1,1]$. So, interpolating the radial function of the skeleton by Legendre polynomials we get some coefficients which will be parametric descriptors of the width (Fig. 4 $4 \mathrm{~d}$ ). Then we can compare the radius functions of the two skeletons just by calculating the Euclidean distance between them using coefficients as coordinates in orthogonal space of Legendre functions. Before interpolating we have to map each skeleton branch into [-1, 1] interval (Fig. 45). 


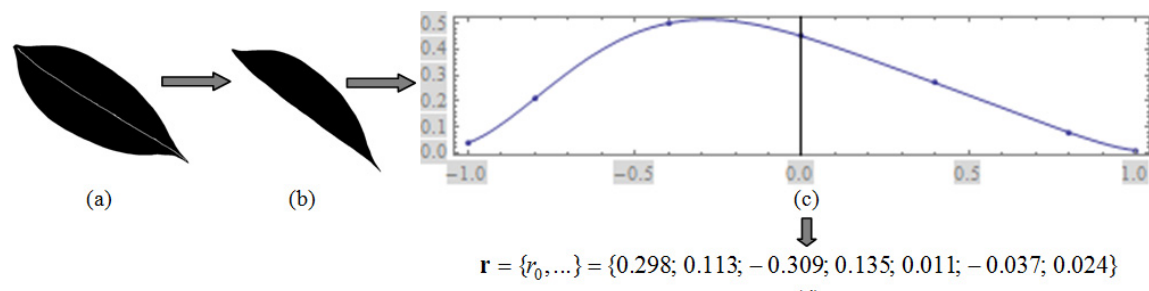

(d)

Fig. 4. The sketch of parametric width description by Legendre polynomials

It is obvious that the number of points with certain width varies in different skeleton branches. Thus we have to reduce the number of Legendre coefficients or augment it by zeros to achieve some predefined number $n$ which shows the proper order of interpolating polynomial for all skeleton ribs. As a result we obtain the vector of fixed length $\mathbf{r}=\left\{r_{0}, \ldots, r_{n}\right\}$ for each skeleton rib. The difference between two radius functions which are interpolated by Legendre polynomials is expressed in the following form:

$$
f\left(\mathbf{r}^{\prime}, \mathbf{r}^{\prime \prime}\right)=\sum_{i=0}^{n}\left(r_{i}^{\prime}-r_{i}^{\prime \prime}\right)^{2} .
$$

The vector of Legendre coefficients is set as an additional component in the skeleton primitive:

$$
\omega=\{l, \alpha, \mathbf{r}\}
$$

Improved node function (2) in pair-wise separable optimization criterion (1) looks as follows

$$
\rho\left(\omega^{\prime}, \omega^{\prime \prime}\right)=\left(l^{\prime}-l^{\prime \prime}\right)^{2}+\left(\alpha^{\prime}-\alpha^{\prime \prime}\right)^{2}+f\left(\mathbf{r}^{\prime}, \mathbf{r}^{\prime \prime}\right) .
$$

\section{Model Experiments}

To proof our assumption artificial data were created. There are three classes of model curves, three in each class. The interval on the axis of abscissas emulates the certain skeleton rib here and the curve emulates the width function. As far as we know only discrete values of radius function of real-world skeleton ribs, we chose several points on model charts which correspond with the following set of arguments $-1.0 ;-0.8 ;-0.6 ;-0.4 ; 0.0 ; 0.4 ; 0.8 ; 0.6 ; 1.0$ (see Fig. 5 a).

Two subsets of arguments were selected to emulate two mesh functions which have the shift related to each other:

$-1.0 ;-0.8 ;-0.4 ; 0.0 ; 0.4 ; 0.8 ; 1.0$ (the first subset),

$-1.0 ;-0.6 ;-0.4 ; 0.0 ; 0.4 ; 0.6 ; 1.0$ (the second subset).

We interpolated those values and have got eighteen vectors of Legendre coefficients, each vector $\mathbf{r}$ has seven components. Pair-wise Euclidean distances 


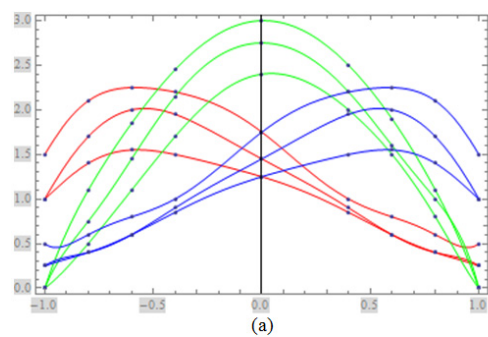

(a)

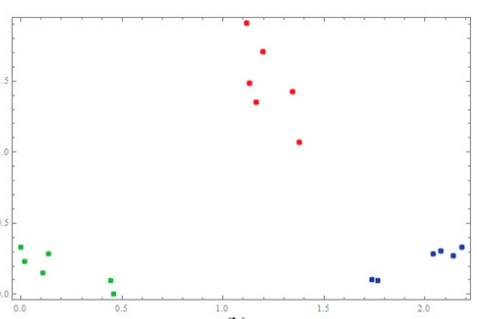

(b)

Fig. 5. (a) Model curves and the set of points for experiments; (b) 2D-visualization of the distances between vectors of Legendre coefficients for the approximation of curves

between curves (presented as vectors of Legendre coefficients) were calculated. The result of the visualization of distance matrix in 2D is shown in Fig. 5b.

It is clear that curves of the same class represented by interpolating vectors are nearer to each other than to the curves of the other classes. There are three clusters in orthogonal Legendre basis. Moreover, width description by Legendre parameters is stable for the curves of the same classes in case of irregular meshes for interpolating functions. Thus, based on our model experiment we can use proposed parametric width description of skeleton ribs for the classification tasks.

\section{Experiments on Real-World Data}

We use the 3-nearest neighbours classifier relying on the dissimilarity function (4) between two objects. We choose the fixed number $(P=10,15,20, \ldots)$ of species representers (patterns) as a training set. Objects left in the dataset are considered as a testing set. The object is referred to some class if the most number of patterns closest to this object belong to that class. In case of equal number of closest patterns from different classes (for example, three nearest neighbours from three different classes) the tested object is classified according to minimum of dissimilarity with a certain pattern. Thus, we achieve the classification decision for the tested objects coinciding with the real or not. Having done this operation several times for randomly chosen patterns and given mean results we can present them in several demonstrable forms.

First, it is the mean number of true classified objects in each class and standard deviation of classification results for five random samples of patterns. In Fig. 6] mean and standard deviation for different number of patterns are presented. Classification rate for each class with the number of patterns $P=45$ is shown in Fig. 7

For more detailed analysis of misclassification it makes sense to examine the non-symmetric contingency table [18. The integer numbers in cells $(i, j)$ mean how many objects of real class $j$ were assigned to the class $i$. From this table we can evaluate different parameters of classifier quality: for example, the interclass dissimilarity and the intraclass variance. 


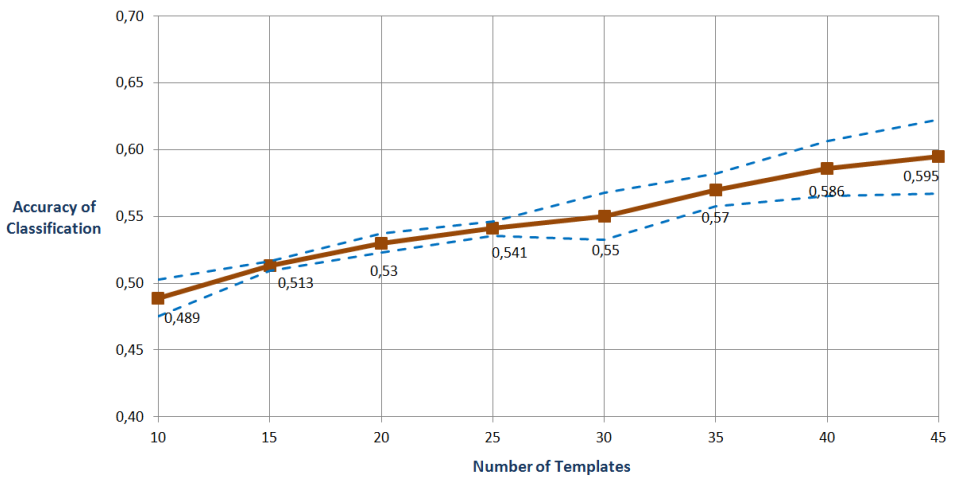

Fig. 6. Mean value (for 5 samplings) of total accuracy and standard deviation of classification results depending on different number of patterns (solid line shows mean value, dash lines show standard deviation)

\begin{tabular}{|c|c|c|c|c|c|c|c|c|c|c|c|c|c|c|c|}
\hline Class & Image & Accuracy & Deviation & Class & Image & Accuracy & Deviation & Class & Image & Accuracy & Deviation & Class & Image & Accuracy & Deviation \\
\hline 1 & 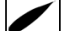 & 0.75 & 0.06 & 9 & & 0.16 & 0.18 & 17 & & 0.87 & 0.06 & 25 & & 0.47 & 0.18 \\
\hline 2 & & 0.7 & 0.06 & 10 & & 0.04 & 0.1 & 18 & & 0.89 & 0.06 & 26 & & 0.8 & 0.13 \\
\hline 3 & & 0.32 & 0.07 & 11 & & 0.16 & 0.09 & 19 & & 0.65 & 0.1 & 27 & & 0.73 & 0.16 \\
\hline 4 & & 0.76 & 0.08 & 12 & & 0.76 & 0.06 & 20 & & 0.94 & 0.06 & 28 & & 0.34 & 0.15 \\
\hline 5 & & 0.77 & 0.07 & 13 & & 0.4 & 0.12 & 21 & & 0.96 & 0.06 & 29 & & 0.48 & 0.07 \\
\hline 6 & 点 & 0.55 & 0.14 & 14 & & 0.66 & 0.04 & 22 & & 0.8 & 0.1 & 30 & & 0.58 & 0.05 \\
\hline 7 & & 0.68 & 0.07 & 15 & & 0.48 & 0.03 & 23 & & 0.12 & 0.1 & 31 & & 0.75 & 0.13 \\
\hline 8 & & 0.83 & 0.2 & 16 & & 0.75 & 0.12 & 24 & & 0.54 & 0.14 & 32 & & 0.35 & 0.15 \\
\hline
\end{tabular}

Fig. 7. Classification rate for each class (number of patterns $P=45$ )

\section{Conclusion}

We have found a novel way to describe shapes of objects based on skeletonization and chain description. Comparing to works of Bai, Klein, Bystrov, Vizilter and others it gives us much richer description of the skeleton representation. Besides the length of the skeleton edge and angle between edges we have introduced Legendre polynomials in order to describe the width of the objects. That gives us a representation of the skeleton as a chain of primitives; each primitive is a vector of length, angle and Legendre coefficients. Based on that representation we calculate pair-wise dissimilarity between shapes. This value is taken into account for dissimilarity classification. We achieve a mean accuracy of about $60 \%$. 
The rate of classification based on shape for different leaf datasets usually isn't much higher than we achieved using our method, e.g. see 3. Moreover, datasets in those researches consist of less species than ours. Such moderate results are mostly explained by the high intra-species variation within some classes and the low inter-species variation between species with ovate leaves. To increase the accuracy they propose to use other modalities such as texture of leaves. In this case the result is raised up to about $80 \%$.

Consequently our classification result is good enough for the leaves recognition task. We will continue our efforts for improving it by additional analyzing our dataset and using inter-class clustering for some challenged species or hierarchical classification. Also we plan to use more powerful classifier, for example, featureless version of SVM 1415].

Acknowledgments. This work was partially supported by the grants of the Russian Fund of Basic Research 12-07-92000, 14-07-00527, 14-07-31271. The authors would like to thank Prof. Leonid Mestetskiy for providing dataset and Prof. Petra Perner for discussions and advice in preparing the text of the paper.

\section{References}

1. Bai, X., Latecki, L.J.: Path similarity skeleton graph matching. IEEE Transactions on Pattern Analysis and Machine Intelligence 30(7), 1282-1292 (2008)

2. Balfer, J., Schöler, F., Steinhage, V.: Semantic Skeletonization for Structural Plant Analysis. Submitted to International Conference on Functional-Structural Plant Models (2013)

3. Beghin, T., Cope, J.S., Remagnino, P., Barman, S.: Shape and texture based plant leaf classification. In: Blanc-Talon, J., Bone, D., Philips, W., Popescu, D., Scheunders, P. (eds.) ACIVS 2010, Part II. LNCS, vol. 6475, pp. 345-353. Springer, Heidelberg (2010)

4. Blum, H.: A transformation for extracting new descriptors of shape. Models for the Perception of Speech and Visual form 19(5), 362-380 (1967)

5. Bystrov, M.Y.: Structural approach application for recognition of binary image skeleton. In: Proceedings of Petrozavodsk State University, vol. 2(115), pp. 76-80 (2011) (in Russian)

6. Demirci, M.F., Shokoufandeh, A., Keselman, Y., Bretzner, L., Dickinson, S.: Object recognition as many-to-many feature matching. International Journal of Computer Vision 69(2), 203-222 (2006)

7. Du, J.X., Huang, D.S., Wang, X.F., Gu, X.: Computer-aided plant species identification (CAPSI) based on leaf shape matching technique. Transactions of the Institute of Measurement and Control 28(3), 275-285 (2006)

8. Jänichen, S., Perner, P.: Aligning concave and convex shapes. In: Yeung, D.-Y., Kwok, J.T., Fred, A., Roli, F., de Ridder, D. (eds.) SSPR 2006 and SPR 2006. LNCS, vol. 4109, pp. 243-251. Springer, Heidelberg (2006)

9. Kadir, A., Nugroho, L.E., Susanto, A., Santosa, P.I.: A comparative experiment of several shape methods in recognizing plants. arXiv preprint arXiv:1110.1509 (2011)

10. Klein, P., et al.: A tree-edit-distance algorithm for comparing simple, closed shapes. In: Proceedings of the Eleventh Annual ACM-SIAM Symposium on Discrete Algorithms. Society for Industrial and Applied Mathematics, pp. 696-704 (2000) 
11. Kushnir, O., Seredin, O.: A pair-wise comparison function for skeleton matching based on primitive sequences alignment. Izvestiya of Tula State University, ser. Technical sciences, Vol. 2. Tula, TSU, pp. 197-207 (2013) (in Russian)

12. Maragos, P.: Pattern spectrum and multiscale shape representation. IEEE Transactions on Pattern Analysis and Machine Intelligence 11(7), 701-716 (1989)

13. Mestetskiy, L., Semenov, A.: Binary Image Skeleton-Continuous Approach. In: VISAPP, vol. (1) (2008)

14. Mottl, V., Seredin, O., Dvoenko, S., Kulikowski, C., Muchnik, I.: Featureless pattern recognition in an imaginary Hilbert space. In: Proceedings of 16th International Conference on Pattern Recognition, vol. 2, pp. 88-91 (2002)

15. Mottl, V., Krasotkina, O., Seredin, O., Muchnik, I.: Kernel fusion and feature selection in machine learning. In: Proceedings of the Eighth IASTED International Conference on Intelligent Systems and Control, ISC 2005, pp. 477-482 (2005)

16. Neuhaus, M., Bunke, H.: Edit distance-based kernel functions for structural pattern classification. Pattern Recognition 39(10), 1852-1863 (2006)

17. Mottl, V.V., et al.: Optimization techniques on pixel neighborhood graphs for image processing. In: Jolion, J.-M., Kropatsch, W.G. (eds.) Graph-Based Representations in Pattern Recognition Computing Supplement, vol. 12, pp. 135-145. Springer, Wien (1998)

18. Perner, P., Zscherpel, U., Jacobsen, C.: A comparison between neural networks and decision trees based on data from industrial radiographic testing. Pattern Recognition Letters 22(1), 47-54 (2001)

19. Reier, I.A.: Plane Figure Recognition Based on Contour Homeomorphism. Pattern Recognition and Image Analysis c/c of raspoznavaniye obrazov i analiz izobrazhenii 11(1), 242-245 (2001)

20. Sebastian, T.B., Benjamin, B.: Curves vs. skeletons in object recognition. Signal Processing 85(2), 247-263 (2005)

21. Sederberg, T.W., Greenwood, E.: A Physically Based Approach to 2-D Shape Blending. Comput. Graphics 26(2), 25-34 (1992)

22. Serra, J.: Image Analysis and Mathematical Morphology. Acad. Press, London (1982)

23. Söderkvist, O.: Computer vision classification of leaves from Swedish trees. Diss. Linköping (2001)

24. Vizilter, Y.V., et al.: Morphological shape comparison based on skeleton representations. Pattern Recognition and Image Analysis 22(3), 412-418 (2012)

25. Wang, C., et al.: An Image Skeletonization Based Tool for Pollen Tube Morphology Analysis and Phenotyping. Journal of Integrative Plant Biology 55(2), 131-141 (2013)

26. Wang, Z., Chi, Z., Feng, D.: Shape based leaf image retrieval. In: IEEE Proceedings of Vision, Image and Signal Processing, vol. 150(1), pp. 34-43 (2003) 